\title{
Article
}

\section{A Note on the Estrada Index of the $A_{\alpha}$-Matrix}

\author{
Jonnathan Rodríguez *(D) and Hans Nina (D)
}

check for

updates

Citation: Rodríguez, J.; Nina, H. A Note on the Estrada Index of the $A_{\alpha}$-Matrix. Mathematics 2021, 9, 811. https://doi.org/10.3390/ math9080811

Academic Editor: János Sztrik

Received: 5 March 2021

Accepted: 5 April 2021

Published: 8 April 2021

Publisher's Note: MDPI stays neutral with regard to jurisdictional claims in published maps and institutional affiliations.

Copyright: (c) 2021 by the authors. Licensee MDPI, Basel, Switzerland. This article is an open access article distributed under the terms and conditions of the Creative Commons Attribution (CC BY) license (https:// creativecommons.org/licenses/by/ $4.0 /)$.
Departamento de Matemáticas, Facultad de Ciencias Básicas, Universidad de Antofagasta, Av. Angamos 601, Antofagasta 1240000, Chile; hans.nina@uantof.cl

* Correspondence: jonnathan.rodriguez@uantof.cl

\begin{abstract}
Let $G$ be a graph on $n$ vertices. The Estrada index of $G$ is an invariant that is calculated from the eigenvalues of the adjacency matrix of a graph. V. Nikiforov studied hybrids of $A(G)$ and $D(G)$ and defined the $A_{\alpha}$-matrix for every real $\alpha \in[0,1]$ as: $A_{\alpha}(G)=\alpha D(G)+(1-\alpha) A(G)$. In this paper, using a different demonstration technique, we present a way to compare the Estrada index of the $A_{\alpha}$-matrix with the Estrada index of the adjacency matrix of the graph $G$. Furthermore, lower bounds for the Estrada index are established.
\end{abstract}

Keywords: Estrada index; $\alpha$-adjacency matrix; adjacency matrix; Laplacian matrix

MSC: 05C50; 15A18

\section{Introduction}

Throughout the paper, we consider $G$ an arbitrary connected graph with the edge set denoted by $\mathcal{E}(G)$ and its vertex set $V(G)=\{1, \ldots, n\}$ with cardinality $m$ and $n$ (order of $G)$, respectively. We say that $G$ is an $(n, m)$-graph. If $e \in \mathcal{E}(G)$ has end vertices $i$ and $j$, then we say that $i$ and $j$ are adjacent, and this edge is denoted by $i j$. For a finite set $U,|U|$ denotes its cardinality. Let $K_{n}$ be the complete graph with $n$ vertices and $\overline{K_{n}}$ its complement.

The adjacency matrix $A(G)$ of the graph $G$ is a symmetric matrix of order $n$ with entries $a_{i j}$, such that $a_{i j}=1$ if $i j \in \mathcal{E}(G)$ and $a_{i j}=0$ otherwise. Denote by $\lambda_{1} \geq \ldots \geq \lambda_{n}$ the eigenvalues of $A(G)$; see [1,2].

The matrix $L(G)=D(G)-A(G)$ is called the Laplacian matrix of $G$ (see [3]), where $D(G)$ is the diagonal matrix of vertex degrees of $G$. We denote the eigenvalues of the Laplacian matrix by $\mu_{1} \geq \mu_{2} \geq \ldots \geq \mu_{n-1} \geq \mu_{n}=0$. A matrix is singular if it has zero as an eigenvalue; otherwise, it is called non-singular. A graph $G$ is said to be non-singular if its adjacency matrix is non-singular.

The Estrada index of the graph $G$ is defined as:

$$
E E(G)=\sum_{i=1}^{n} e^{\lambda_{i}}
$$

This spectral quantity was put forward by E. Estrada [4] in the year 2000. Many chemical and physical applications have been found, including quantifying the degree of folding of long-chain proteins [5-7] and complex networks [8-11]. The mathematical properties of this invariant can be found in, e.g., [12-16].

De la Peña et al. in [17], with respect to Estrada index, showed the following.

Theorem 1 ([17]). Let $G$ be an $(n, m)$-graph. Then, the Estrada index of $G$ is bounded as:

$$
\sqrt{n^{2}+4 m} \leq E E(G) \leq n-1+e^{\sqrt{2 m}}
$$

Equality on both sides of (1) is attained if and only if $G$ is isomorphic to $\overline{K_{n}}$. 
In [18], Bamdad proved the following result.

Theorem 2 ([18]). Let $G$ be an $(n, m)$-graph with $t$ triangles. Then:

$$
E E(G) \geq \sqrt{n^{2}+2 m n+2 n t} .
$$

Equality holds if and only if $G$ is the empty graph $\overline{K n}$.

Denote by $M_{k}=M_{k}(G)$ the $k$-th spectral moment of the graph $G$, i.e.,

$$
M_{k}=\sum_{i=1}^{n}\left(\lambda_{i}\right)^{k},
$$

then, we can write the Estrada index as:

$$
E E(G)=\sum_{k=0}^{\infty} \frac{M_{k}}{k !} .
$$

In [1], for an $(n, m)$-graph $G$, the authors proved that:

$$
M_{0}=n, M_{1}=0, M_{2}=2 m, M_{3}=6 t,
$$

where $t$ is the number of triangles in $G$.

Remark 1. Notice that we can obtain a lower bound for the Estrada index considering (3) and (4) by:

$$
\begin{aligned}
E E(G) & =\sum_{k=0}^{\infty} \frac{M_{k}}{k !} \\
& =M_{0}+M_{1}+\frac{M_{2}}{2 !}+\frac{M_{3}}{3 !}+\sum_{k=4}^{\infty} \frac{M_{k}}{k !} \\
& \geq M_{0}+M_{1}+\frac{M_{2}}{2 !}+\frac{M_{3}}{3 !} \\
& =n+m+t .
\end{aligned}
$$

Therefore, we demonstrate the following result.

Theorem 3. Let $G$ be an $(n, m)$-graph with $t$-triangles. Then:

$$
E E(G) \geq n+m+t .
$$

Equality holds if and only and $G$ is isomorphic to $\overline{K_{n}}$.

Remark 2. Here, we show that the bound in Theorem 3 improves the bounds in Theorems 1 and 2. Suffice it to show that:

$$
\sqrt{n^{2}+4 m} \leq n+m+t \quad \Rightarrow \quad 4 m \leq m^{2}+t^{2}+2(n m+n t+m t) .
$$

and:

$$
\sqrt{n^{2}+2 n m+2 n t} \leq n+m+t \Rightarrow 0 \leq m^{2}+t^{2}+2 m n .
$$

In [19], V. Nikiforov studied hybrids of $A(G)$ and $D(G)$ and defined the $A_{\alpha}$-matrix for every real $\alpha \in[0,1]$ as:

$$
A_{\alpha}(G)=\alpha D(G)+(1-\alpha) A(G),
$$


with $\rho_{1}, \rho_{2}, \ldots, \rho_{n}$ the eigenvalues of $A_{\alpha}$.

The Estrada index of the $A_{\alpha}$-matrix of graph $G$ is defined as

$$
E E_{\alpha}(G)=\sum_{i=1}^{n} e^{\rho_{i}}
$$

Note that the $A_{\alpha}$-matrix can be written as follows:

$$
A_{\alpha}(G)=\alpha(L(G))+A(G) .
$$

Given a matrix $M$, we denote by $\partial_{i}(M)$ the $i$-th eigenvalue in descending order of matrix $M$. The following result, due to Weyl, can be found in [20].

Theorem 4 ([20]). Let $A$ and $B$ be two Hermitian matrices of order $n$, and let $1 \leq i, j \leq n$. If $C=A+B$ is a matrix, then,

(i) $\partial_{i}(A)+\partial_{j}(B) \leq \partial_{i+j-n}(C)$, if $i+j \geq n+1$;

(ii) $\partial_{i}(A)+\partial_{j}(B) \geq \partial_{i+j-1}(C)$, if $i+j \leq n+1$.

Equality holds if and only if there exists a unit vector that is an eigenvector of each of the three eigenvalues involved.

Notice that if $j=n$ and $j=1$ in Weyl's inequality; we can write:

$$
\partial_{i}(A)+\partial_{n}(B) \leq \partial_{i}(A+B) \leq \partial_{i}(A)+\partial_{1}(B) .
$$

Applying the inequality (6) to the matrix in (5), i.e., considering $A=A(G)$ and $B=\alpha L(G)$, we have the following inequalities.

$$
\partial_{i}(A(G))+\alpha \partial_{n}(L(G)) \leq \partial_{i}\left(A_{\alpha}(G)\right) \leq \partial_{i}(A(G))+\alpha \partial_{1}(L(G)) .
$$

In 1985, Anderson et al. [3] obtained the following upper bound for the Laplacian matrix.

Lemma 1. [3] If $G$ is a graph of order $n$, then:

$$
\mu_{1}(L(G)) \leq n .
$$

Equality holds if and only if $\bar{G}$ is disconnected.

Considering the above Lemma 1 , the inequality $(7)$, and $\partial_{n}(L(G))=\mu_{n}=0$, we have:

$$
\partial_{i}(A(G)) \leq \partial_{i}\left(A_{\alpha}(G)\right) \leq \partial_{i}(A(G))+n \alpha .
$$

Applying the exponent function and sum over $i=1, \ldots, n$; we have:

$$
\sum_{i=1}^{n} e^{\partial_{i}(A(G))} \leq \sum_{i=1}^{n} e^{\partial_{i}\left(A_{\alpha}(G)\right)} \leq e^{n \alpha} \sum_{i=1}^{n} e^{\partial_{i}(A(G))} .
$$

Hence, we get the following results.

$$
E E(G) \leq E E\left(A_{\alpha}(G)\right) \leq e^{n \alpha} E E(G) .
$$

As a consequence of the inequality (10), Lemma 3, and Theorem 1, we have the following result. 
Theorem 5. Let $G$ be an $(n, m)$-graph. Then, the Estrada index of $A_{\alpha}$ is bounded as:

$$
\sqrt{n^{2}+4 m} \leq E E\left(A_{\alpha}(G)\right) \leq e^{n \alpha}\left(n-1+e^{\sqrt{2 m}}\right)
$$

and:

$$
E E\left(A_{\alpha}(G)\right) \geq n+m+t .
$$

The equality case on both inequalities is attained if and only if $\alpha=0$ and $G$ is isomorphic to $\overline{K_{n}}$.

In this paper, new lower bounds for the Estrada index are established. Considering Theorem 3 and the results previously shown, we allow obtaining new lower bounds for the Estrada index of the $A_{\alpha}$-matrix.

\section{Estrada Index and Energy}

In this section, in order to obtain new lower bounds to approximate the value of the Estrada index of the $A_{\alpha}$-matrix, new lower bounds are established for the Estrada index in relation to the energy of the $G$ graph.

The energy of a graph $G$ was defined by Ivan Gutman in 1978 [21] as:

$$
E(G)=\sum_{i=1}^{n}\left|\lambda_{i}\right|
$$

The energy of a graph $G$ is studied in mathematical chemistry and used to approximate the total $\pi$-electron energy of a molecule. Eventually, it was recognized that the interest in this graph invariant goes far beyond chemistry; see the recent papers [22-26] and the references cited therein.

In [27], Koolen and Moulton showed that the following relation holds for all graphs $G$

$$
E(G) \leq \lambda_{1}+\sqrt{(n-1)\left(2 m-\lambda_{1}^{2}\right)}
$$

For all $(n, m)$-graphs $G$ connected and nonsingular, Das et al. in [28] proved the following relation holds:

$$
E(G) \geq \lambda_{1}+(n-1)+\ln (\operatorname{det} A)+\ln \left(\lambda_{1}\right),
$$

then using the inequality $2 m / n \leq \lambda_{1}$ in (13) and (14), they obtained the following upper and lower bounds, respectively:

$$
E(G) \leq \frac{2 m}{n}+\sqrt{(n-1)\left(2 m-\left(\frac{2 m}{n}\right)^{2}\right)}
$$

and:

$$
E(G) \geq \frac{2 m}{n}+(n-1)+\ln |\operatorname{det}(A)|+\ln \left(\frac{2 m}{n}\right) .
$$

Theorem 6. Let $G$ be an $(n, m)$-graph with $k$ non-negative eigenvalues. Then:

$$
E E(G) \geq \frac{E(G)}{2}+e^{\left(\frac{2 m}{n}\right)}+(k-1)-\frac{2 m}{n} .
$$

Equality holds in (15) if and only if $G$ is isomorphic to $\overline{K_{n}}$.

Proof. Let $x \geq 0$, and consider the following function:

$$
g(x)=-1-x+e^{x}
$$


the equality holds if and only if $x=0$. It is straightforward to show that function $g(x)$ is increasing in $[0,+\infty)$. Then, $g(x) \geq g(0)$, implying that:

$$
x \leq e^{x}-1, \quad x \geq 0 .
$$

Note that, as $A(G)$ is a symmetric matrix with zero trace, these eigenvalues are real with the sum equal to zero, i.e.,

$$
\lambda_{1} \geq \cdots \geq \lambda_{n}
$$

and:

$$
\lambda_{1}+\cdots+\lambda_{n}=0 .
$$

Then, by the definition of the energy join to (18) and (19), we have:

$$
\frac{E(G)}{2}=\sum_{\lambda_{i}>0} \lambda_{i}^{+}=-\sum_{\lambda_{i}<0} \lambda_{i}^{-} .
$$

Suppose that $A(G)$ have $k$ non-negative eigenvalues, then using (20) and (17), we obtain:

$$
\begin{aligned}
\frac{E(G)}{2} & =\sum_{i=1, \lambda_{i} \geq 0}^{k} \lambda_{i} \\
& =\lambda_{1}+\sum_{i=2, \lambda_{i} \geq 0}^{k} \lambda_{i} \\
& \leq \lambda_{1}+\sum_{i=2, \lambda_{i} \geq 0}^{k}\left(e^{\lambda_{i}}-1\right) \\
& =\lambda_{1}-(k-1)+\sum_{i=1, \lambda_{i} \geq 0}^{k} e^{\lambda_{i}}-\left(e^{\lambda_{1}}\right) \\
& \leq \lambda_{1}-(k-1)+\sum_{i=1, \lambda_{i} \geq 0}^{k} e^{\lambda_{i}}+\sum_{i=k+1, \lambda_{i}<0}^{n} e^{\lambda_{i}}-e^{\lambda_{1}} \\
& =\lambda_{1}-(k-1)+\sum_{i=1}^{n} e^{\lambda_{i}}-e^{\lambda_{1}} .
\end{aligned}
$$

Thereby, considering $\lambda_{1} \geq \frac{2 m}{n}$, we obtain the first result.

Suppose now that the equality holds. From the equality in (16), we get $\lambda_{1}=\ldots=$ $\lambda_{n}=0$. Then, $k=n$. Therefore, $G$ is isomorphic to $\overline{K_{n}}$. Note that if $G$ is equal to $\overline{K_{n}}$, it is easy to check that the equality in (15) holds.

As a consequence of the above theorem and the lower bound due to Das et al. in (14), we obtain the following result.

Corollary 1. Let $G$ be a connected non-singular graph of order $n$ with $k$ strictly positive eigenvalues. Then:

$$
E E(G) \geq \frac{1}{2}\left(n-1+\ln (\operatorname{det}(A(G)))+\ln \left(\lambda_{1}\right)\right)+e^{\lambda_{1}}+(k-1)-\frac{\lambda_{1}}{2} .
$$

\section{Comparison and Conclusions}

In this section, in order to show that our results improve the existing results in the literature, we present some computational experiments to compare our new lower bounds with the lower bounds existing in the literature for connected graphs. For comparison reasons, we consider the explicit values of the eigenvalues of the mentioned graphs. In the following table, the real value of the Estrada index of some graphs is compared with the approximate 
values obtained by applying Theorem 3 (Thm.3) and Theorem 6 (Thm.6) obtained in this paper together with some existing results in the literature, for example Theorem 1 (Thm.1) in [17], Theorem 13 in [23] (Thm.13), and Theorem 2 (Thm.2) in [18].

\begin{tabular}{ccccccc}
\hline Graph & EE(G) & Thm 1 & Thm 2 & Thm 13 & Thm 3 & Thm 6 \\
\hline Herschel & 45.195 & 22.738 & 13.892 & 26.5084 & 29.000 & $\mathbf{3 2 . 9 8 8}$ \\
\hline Heawood & 46.176 & 28.000 & 16.733 & 30.1952 & 35.000 & 34.571 \\
\hline Petersen & 34.218 & 20.000 & 12.649 & 21.2832 & 25.000 & $\mathbf{3 0 . 0 8 6}$ \\
\hline Grötzsch & 55.619 & 23.685 & 14.177 & 29.0915 & 33.000 & $\mathbf{4 8 . 0 1 9}$ \\
\hline$K_{4}$ & 21.189 & 9.7980 & 6.3246 & 10.6829 & 14.000 & $\mathbf{2 0 . 0 8 6}$ \\
\hline$K_{5}$ & 56.070 & 15.000 & 8.0623 & 22.8344 & 25.000 & $\mathbf{5 4 . 5 9 8}$ \\
\hline$S_{5}$ & 10.524 & 8.0623 & 6.4031 & 8.0103 & $\mathbf{9 . 0 0 0}$ & 8.3530 \\
\hline$S_{6}$ & 13.463 & 9.7980 & 7.4833 & 9.7098 & $\mathbf{1 1 . 0 0 0}$ & 9.8639 \\
\hline$C_{4}$ & 9.5244 & 6.9282 & 5.6569 & 7.2896 & 8.000 & $\mathbf{9 . 3 8 9 1}$ \\
\hline$C_{5}$ & 11.503 & 8.6603 & 6.7082 & 8.7768 & 10.000 & $\mathbf{1 0 . 6 2 5}$ \\
\hline$P_{4}$ & 7.6479 & 6.3246 & 5.2915 & 6.3059 & $\mathbf{7 . 0 0 0}$ & 6.2217 \\
\hline$P_{5}$ & 9.941 & 8.0620 & 6.4031 & 7.9106 & $\mathbf{9 . 0 0 0}$ & 8.083 \\
\hline$K_{2,3}$ & 14.669 & 9.2195 & 7.000 & 9.9628 & 11.000 & $\mathbf{1 4 . 0 7 3}$ \\
\hline
\end{tabular}

Analyzing the above examples, we observe the following:

- In all our test cases, our lower bounds are better than existing bounds in the literature. Furthermore, we confirm Remark 2.

- Considering the results obtained in this paper, a possible way is to apply them to digraphs, which have seen relevant interest recently among researchers.

Author Contributions: Conceptualization, J.R. and H.N.; methodology, J.R.; software, J.R.; validation, J.R. and H.N.; formal analysis, H.N.; investigation, J.R.; resources, J.R and H.N.; writingoriginal draft preparation, J.R.; writing—review and editing, J.R.; visualization, H.N.; supervision, J.R. All authors have read and agreed to the published version of the manuscript.

Funding: J. Rodríguez Z. was supported by the MINEDUC-UA project, code ANT-1899, funded by the Initiation Program in Research - Universidad de Antofagasta, INI-19-06, and MATHAMSUDFLN-CheGraTA, código 21-MATH-05. Hans Nina was partially supported by Comisión Nacional de Investigación Científica y Tecnológica, Grant FONDECYT 11170389, Chile, and Universidad de Antofagasta, Antofagasta, Chile, Grant UA INI-17-02.

Institutional Review Board Statement: Not applicable.

Informed Consent Statement: Not applicable.

Data Availability Statement: Not applicable.

Conflicts of Interest: The authors declare no conflict of interest.

\section{References}

1. Cvetković, D.; Doob, M.; Sachs, H. Spectra of Graphs Theory and Application; Academic Press: New York, NY, USA, 1980.

2. Cvetković, D.; Rowlinson, P.; Simić, S. An Introduction to the Theory of Graph Spectra; Cambridge University Press: Cambridge, UK, 2010.

3. Anderson, W.N.; Morley, T.D. Eigenvalues of the Laplacian of a graph. Linear Multilinear Algebra 1985, 18, 141-145. [CrossRef]

4. Estrada, E. Characterization of 3D molecular structure. Chem. Phys. Lett. 2000, 319, 713-718. [CrossRef]

5. Estrada, E. Characterization of the amino acid contribution to the folding degree of proteins. Proteins 2004, 54, 727-737. [CrossRef] [PubMed]

6. Gutman, I.; Furtula, B.; Glisic, B.; Markovic, V.; Vesel, A. Estrada index of acyclic molecules. Indian J. Chem. 2007, 46A, 723-728.

7. Gutman, I.; Radenković, S.; Furtula, B.; Mansour, T.; Schork, M. Relating Estrada index with spectral radius. J. Serb. Chem. Soc. 2007, 72, 1321-1327. [CrossRef] 
8. Estrada, E.; Rodríguez-Velázquez, J.A. Subgraph centrality in complex networks. Phys. Rev. E 2005 71, 056-103. [CrossRef] [PubMed]

9. Estrada, E.; Rodríguez-Velázquez, J.A. Spectral measures of bipartivity in complex networks. Phys. Rev. E 2005, 71, 046-105. [CrossRef]

10. Shang, Y. Biased edge failure in scale-free networks based on natural connectivity. Indian J. Phys. 2012, 86, 485-488. [CrossRef]

11. Shang, Y. Random lifts of graphs: Network robustness based on the Estrada index. Appl. Math. E-Notes 2012, $12,53-61$.

12. Khosravanirad, A. A lower bound for Laplacian Estrada index of a graph. MATCH Commun. Math. Comput. Chem. 2013, 70, 175-180.

13. Gutman, I.; Deng, H.; Radenković, S. The Estrada index: An updated survey. In Selected Topics on Applications of Graph Spectra; Cvetković, D., Gutman, I., Eds.; Matematički Institut SANU: Beograd, Serbia, 2011; pp. 155-174.

14. Rodríguez, J. A note on new bounds for the Estrada Index. Linear Algebra Appl. 2019, 580, 121-127. [CrossRef]

15. Zhou, B. On Estrada index. MATCH Commun. Math. Comput. Chem. 2008, 60, 485-492.

16. Shang, Y. Lower bounds for the Estrada index of graphs. Electron. J. Linear Algebra 2012, 23, 664-668. [CrossRef]

17. De la Peña, J.A.; Gutman, I.; Rada, J. Estimating the Estrada index. Linear Algebra Its Appl. 2007, 427, 70-76. [CrossRef]

18. Bamdad, H. New Lower Bounds for Estrada Index. Bull. Malays. Math. Sci. Soc 2016, 39, 683-688. [CrossRef]

19. Nikiforov, V. Merging the A and Q-spectral theories. Appl. Anal. Discret. Math. 2017, 11, 81-107. [CrossRef]

20. Horn, R.; Johnson, C. Matrix Analysis; Cambridge University Press: Cambridge, UK, 1985.

21. Gutman, I. The energy of a graph. Ber. Math. Stat. Sekt. Forschungszentrum Graz 1978, 427, 1-22.

22. Gutman, I.; Filipovski, S.; Jajcay, R. Variations on McClellands bound for graph energy. Discret. Math. Lett. 2020, 3, 57-60.

23. Yang, Y.; Sun, L.; Bu, C. A Note on Some Bounds of the $\alpha$-Estrada Index of Graphs. Adv. Math. Phys. 2020, 1-11. doi:10.1155/2020/3972789. [CrossRef]

24. Zhou, Q.; Wong, D.; Sun, D. A lower bound for graph energy. Linear Multilinear Algebra. 2020, 68, 1624-1632. [CrossRef]

25. Gutman, I.; Ramane, H.S. Research on Graph Energies in 2019. MATCH Commun. Math. Comput. Chem. 2020, 84, 277-292.

26. Akbari, S.; Ghodrati, A.H.; Hosseinzadeh, M.A. Some lower bounds for the energy of graphs. Linear Algebra Its Appl. 2020, 591, 205-214. [CrossRef]

27. Koolen, J.H.; Moulton, V. Maximal energy graphs. Adv. Appl. Math. 2001, 26, 47-52. [CrossRef]

28. Das, K.; Mojallal, S.A.; Gutman, I. Improving McClellands lower bound for energy. MATCH Commun. Math. Comput. Chem. 2013, $70,663-668$. 